\title{
Parameters of galactic early B supergiants
}

\section{The influence of the wind on the interstellar extinction determination}

\author{
M. Kraus ${ }^{1}$, M. Borges Fernandes ${ }^{2,3}$, and J. Kubát ${ }^{1}$ \\ 1 Astronomický ústav, Akademie věd České republiky, Fričova 298, 25165 Ondřejov, Czech Republic \\ e-mail: [kraus; kubat]@sunstel.asu.cas.cz \\ 2 UMR 6525 H. Fizeau, Univ. Nice Sophia Antipolis, CNRS, Observatoire de la Côte d'Azur, Av. Copernic, 06130 Grasse, France \\ e-mail: Marcelo.Borges@obs-azur.fr \\ 3 Royal Observatory of Belgium, Ringlaan 3, 1180 Brussels, Belgium
}

Received 3 June 2008 / Accepted 16 February 2009

\begin{abstract}
Context. The interstellar extinction, $E(B-V)$, of OB supergiants is usually derived from the observed color index, $(B-V)$, in comparison with the intrinsic one. This method works properly only if the stellar wind does not influence the optical continuum emission. Over the past years, many OB supergiants have been found to have wind-velocity distributions with rather high $\beta$ values; i.e., their winds are only slowly accelerating, resulting in relative density enhancements within the wind-continuum forming region. It has been found that these kinds of winds might indeed influence the total continuum emission even at optical wavelengths. Aims. We investigate the influence of the wind on the observed color indices of OB supergiants, in order to distinguish between interstellar and circumstellar extinction contributions.

Methods. We first tested the influence of the wind on the $U B V$ band fluxes of a model $\mathrm{O}$ supergiant star with artificial winds of different $\beta$ values and a fixed interstellar extinction value. From the $U B V$ magnitudes of these systems, the apparent interstellar extinction was then derived by calculating the values of $E(B-V)$, from the $(B-V)$ as well as from the $(U-B)$ color index. Then we turned to a sample of galactic early-type B supergiants for which the stellar and wind parameters are known. All except one of these stars have $\beta$ values higher than 1.0, which makes this set the most suitable one for our investigation. We calculated each star's wind contribution to the $B V$ band fluxes. The observed magnitudes were corrected for the wind contributions, and the interstellar extinctions were derived and compared with those derived purely from the $(B-V)$ color index.

Results. From our model supergiant, we find that with increasing $\beta$ the wind starts to influence the observable color indices. The wavelength dependence of the wind contribution is thereby different from that of the interstellar extinction contribution. Thus, the interstellar extinction values derived from the two color indices of our reddened star plus wind systems disagree. This effect is stronger for higher $\beta$. In addition, $E(B-V)$ derived from the $(B-V)$ color index always overestimates the real interstellar extinction. This trend is also found for the investigated B supergiant sample. Consequently, the luminosities of these stars are systematically overestimated. Our wind model always computes lower limits to the real wind contributions. This means that the real interstellar extinction values, hence the stellar luminosities of the studied B supergiant sample, might well be lower.
\end{abstract}

Key words. stars: early-type - stars: fundamental parameters - supergiants - stars: winds, outflows

\section{Introduction}

The interstellar extinction is one of the most important astrophysical parameters. It is often derived from the observed color index of stars, usually from $B-V$, in comparison with the intrinsic color index, if the spectral type of the star is known. This method is reliable, as long as no additional component (like a wind or disk) adds to the total observable flux in the $B$ and $V$ bands. For typical OB supergiants with their winds of only moderate mass-loss rates, this was thought to be the case.

In the past few years, a lot of effort has been made to determine the stellar and wind parameters of $\mathrm{OB}$ supergiants within and beyond the Milky Way with high accuracy. It thereby turned out that many B-type supergiant stars seem to have wind velocity distributions with rather high $\beta$ values, extending up to about 3.5 (see e.g. Evans et al. 2004; Trundle et al. 2004; Trundle \& Lennon 2005; Crowther et al. 2006)

Waters \& Lamers (1984) were the first to investigate the influence of the wind velocity distribution on the total continuum emission in more detail. They found that a high $\beta$ can lead to an enhanced emission. These studies were, however, restricted to the radio and infrared ranges, where the thermal wind in form of free-free and free-bound emission is known to play the dominant role. Recently, Kraus et al. (2008a,b) have shown that, for OB supergiants with high $\beta$ values, the wind emission can influence the total continuum even at optical wavelengths. This occurs because, for $\beta>1.0$, the density within the innermost $\sim 2 R_{*}$ is strongly enhanced, compared to a wind with $\beta=1.0$. This wind region of enhanced density coincides exactly with the formation region of the optical wind continuum. Any density enhancement close to the star will, therefore, immediately result in an enhanced thermal wind emission at optical wavelengths.

These new findings of the wind influence at optical wavelengths thus raise the question of whether the wind can contaminate the observable $U B V$ band fluxes further such that the interstellar extinction values derived from the color indices are influenced as well. Such an influence has recently been found for the low-luminosity supergiant star AzV 172, for which the interstellar extinction, hence the stellar luminosity, turned out to be overestimated if the circumstellar extinction due to the wind 
is not properly taken into account (Kraus et al. 2008c). This paper therefore aims to provide a detailed investigation of the wind influence on the $U B V$ band fluxes and consequently on the observable color indices of OB supergiants having winds with especially high $\beta$ values.

The structure of the paper is as follows. We first investigate the influence of the wind (for varying mass-loss and $\beta$ parameters) to the observable $U B V$ band fluxes and, therefore, to the apparent interstellar extinction that results from the comparison of the observed to the intrinsic color indices (Sect. 2). Then, we present our sample of early B supergiant stars in Sect. 3. In Sect. 4 we describe our calculations of the interstellar extinction taking the wind contributions into account. The resulting extinction values are further compared to those received purely from the observed $(B-V)$ color index. In Sect. 5 we discuss the wind contribution and its importance, as well as the reliability of our simplified wind calculations. Other consequences of the revised interstellar extinction values are mentioned and discussed. Our results are summarized in Sect. 6.

\section{Interstellar and circumstellar extinction}

To study the influence of winds with high $\beta$ values on the observable color indices, we first calculated the emission of a model star at $U B V$ wavelengths and determine its intrinsic color indices $(U-B)_{0}$ and $(B-V)_{0}$. This is done by using the code of Kubát (2003, and references therein), which is suitable for the computation of non-LTE spherically-symmetric model atmospheres in hydrostatic and radiative equilibrium. The stellar continuum emission of a hydrogen plus helium atmosphere is calculated by adopting the following set of stellar parameters, which approximately corresponds to an $\mathrm{O} 7$ supergiant, based on the calibration of Martins et al. (2005): $T_{\text {eff }}=33000 \mathrm{~K}, R_{*}=17.2 R_{\odot}$, $\log L_{*} / L_{\odot}=5.5$, and $\log g=3.4$. In addition, the star is placed to an arbitrary distance of $1 \mathrm{kpc}$.

With this set of parameters, we computed the stellar flux at the wavelengths of the $U B V$ bands, converted them into magnitudes based on the calibration fluxes of Bessell (1979) for a zero magnitude star, and derived the intrinsic color indices. For our model supergiant, we thus find

$$
(U-B)_{0}=-1.063 \text {, }
$$$$
(B-V)_{0}=-0.263 \text {. }
$$

The stellar fluxes are then distance-diluted and reddened with an arbitrary interstellar extinction value of $A_{V}=0.62$, corresponding to $E(B-V)=0.20$ for $R=A_{V} / E(B-V)=3.1$. The observable reddened fluxes are given by

$F_{\lambda}=F_{\lambda}^{*} \exp \left\{-0.4 \ln (10) \frac{A_{\lambda}}{A_{V}} A_{V}\right\}$.

The parameters $A_{\lambda} / A_{V}$ result from the mean interstellar extinction curve (see e.g. Cardelli et al. 1989; Mathis 1990), and $F_{\lambda}^{*}$ is the unreddened stellar flux. Conversion into magnitudes delivers the following observable color indices

$(U-B)=-0.908$,

$(B-V)=-0.063$.

From these, the interstellar extinction value $E(B-V)$ can be derived from either the $(U-B)$ or the $(B-V)$ color indices, via

$E(B-V)=\frac{1}{0.77}\left[(U-B)-(U-B)_{0}\right]=0.20$
$E(B-V)=(B-V)-(B-V)_{0}=0.20$.

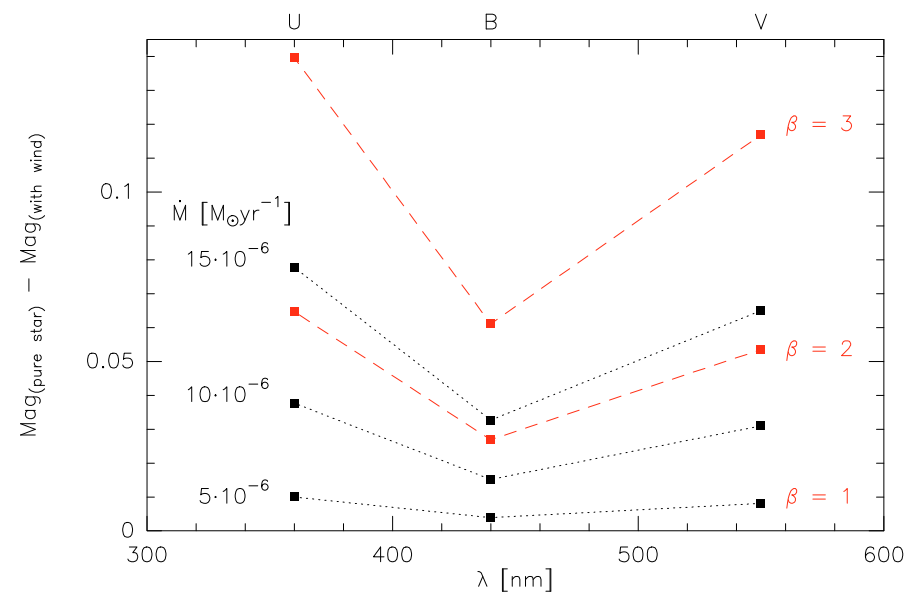

Fig. 1. Brightening of the $U B V$ photometric data due to the presence of high density winds. The dotted lines represent wind models with fixed $\beta=1.0$ and varying mass-loss rates, the dashed ones for a fixed massloss rate of $\dot{M}=5 \times 10^{-6} M_{\odot} \mathrm{yr}^{-1}$ and varying $\beta$ values.

The former relation is taken from Leitherer \& Wolf (1984). Both relations give identical values for $E(B-V)$, which agree with the input value.

Next, we included the stellar wind. The star plus wind system was calculated using the model of Kraus et al. (2008a), which treats the system in the core-halo approximation. This means that we can calculate the observable flux at any wavelength via

$F_{\lambda}=\left(F_{\lambda}^{*} \mathrm{e}^{-\tau_{\lambda}}+F_{\lambda}^{\text {wind }}\right) \mathrm{e}^{-0.4 \ln (10) \frac{A_{\lambda}}{A_{V}} A_{V}}$

where $F_{\lambda}^{\text {wind }}$ is the wind flux due to free-free and free-bound processes in the wind zone, and $\tau_{\lambda}$ is the optical depth of the wind. It is given by the line-of-sight integral from the stellar surface to the outer edge of the wind zone (which is set to infinity) over the wind absorption coefficient. For details on the calculations and a discussion about the influence of the assumptions on the results, we refer to Kraus et al. (2008a). The wind model is, however, such that we always calculate a lower limit of the wind emission.

To investigate the influence of the wind to the total continuum emission, we first neglected any interstellar extinction, but calculate the $U B V$ band fluxes for winds with (i) fixed value of $\beta=1.0$ and mass-loss rates ranging from 5 to $15 \times 10^{-6} M_{\odot} \mathrm{yr}^{-1}$ and (ii) fixed mass-loss rate of $5 \times 10^{-6} M_{\odot} \mathrm{yr}^{-1}$ and $\beta$ values ranging from 1.0 to 3.0. We further fixed the terminal velocity at $v_{\infty}=1400 \mathrm{~km} \mathrm{~s}^{-1}$, and we kept the electron temperature at a constant value of $T_{\mathrm{e}}=25760 \mathrm{~K}$. This is a reasonable assumption as shown and discussed in Kraus et al. (2008a). The resulting fluxes from the star plus wind systems are converted into magnitudes, which are then compared to the intrinsic (i.e. pure) stellar magnitudes. This comparison is shown for the set of wind models in Fig. 1, where we plot the difference between the intrinsic stellar magnitudes and the magnitudes of the star with wind systems obtained in the three photometric bands.

Obviously, as soon as a wind is included, the star brightens in all bands, with the strongest effect in the $U$ and $V$ bands, while the $B$ band is only marginally affected. Also obvious is that a $\beta$ value of 3.0 has a much stronger effect than an increase in massloss rate (hence in wind density) by a factor of 3 . This can be understood by inspecting the density distribution in the winds with different $\beta$ values (Fig. 2). As shown by Kraus et al. (2008a), the optical continuum of the wind is generated within the innermost $2 R_{*}$. In this region, the wind with $\beta=3.0$ remains at much 

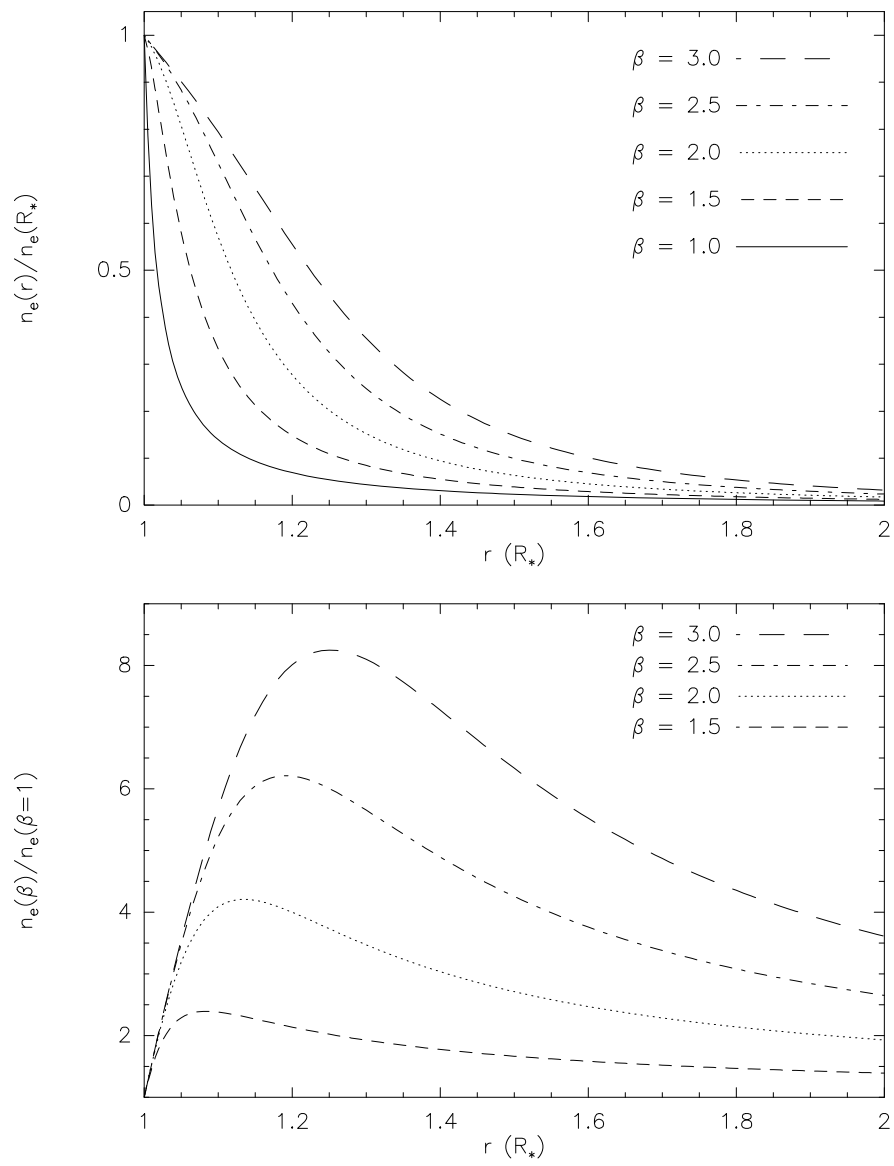

Fig. 2. Density distribution in the wind continuum forming regions, i.e., within $2 R_{*}$, for winds with different $\beta$ values, normalized to the density on the stellar surface (top panel), and for winds with $\beta>1.0$ with respect to the wind with $\beta=1.0$ (bottom panel).

higher densities due to the slow wind acceleration (top panel of Fig. 2). The difference to the wind with $\beta=1.0$ exceeds a factor of 8 at a distance of about $1.2 \ldots 1.3 R_{*}$ and is still almost 4 times higher at $2 R_{*}$ (bottom panel of Fig. 2). This huge difference in density causes the strong wind contribution to the optical continuum, especially in the $U$ and $V$ bands, resulting in modified observable colors of the star, even in the absence of any interstellar extinction. Another consequence of the wind, which is also visible in Fig. 1 and which will be confirmed and discussed further in Sect. 5.3 below, is the apparent higher stellar luminosity, which results from the $V$ band flux as long as no correction of the photometric data, accounting for the wind contamination, is taken into account.

Next, we included the interstellar extinction, which we fixed at $A_{V}=0.62$, i.e., at $E(B-V)=0.20$. We first calculated a reference wind model adopting $\beta=1.0$, and a mass-loss rate of $\dot{M}=5 \times 10^{-6} M_{\odot} \mathrm{yr}^{-1}$. The reddened and distance diluted fluxes, calculated with Eq. (8) and converted into magnitudes, deliver as observable color indices for this star plus wind system values of $(U-B)=-0.914$ and $(B-V)=-0.059$. If these values are then used to derive the interstellar extinction from the two relations (6) and (7), we find $E(B-V)=0.194$ from the former and $E(B-V)=0.204$ from the latter. These two values, though still in fairly good agreement, already show that, even for stars with winds of moderate mass-loss rates and with a $\beta$ value of 1.0 , the wind starts to influence the total emission at the $U B V$ bands.
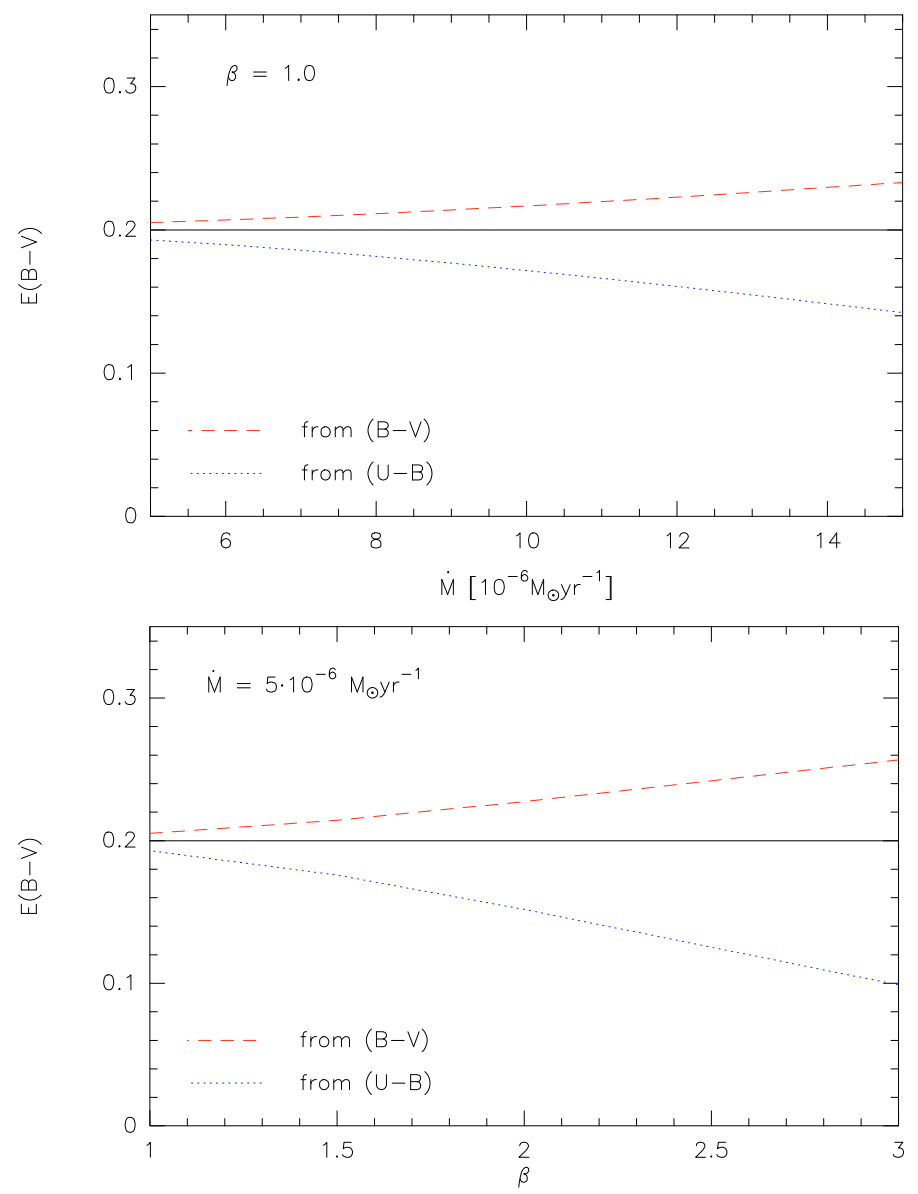

Fig. 3. Apparent interstellar extinction value of a star plus wind system, resulting from different (increasing) mass-loss rates (top) or different $\beta$ values (bottom). The input interstellar extinction is fixed at $E(B-V)=$ 0.2 (shown as solid lines). The stellar model corresponds approximately to an O7 supergiant, based on the calibration of Martins et al. (2005). The two curves give the interstellar extinction as derived from either the color index $(B-V)$ or $(U-B)$.

We next consider the influence of increasing mass-loss rate for a fixed $\beta$ value of 1.0, as well as the influence of increasing $\beta$ for a fixed mass-loss rate. In both cases, the input interstellar extinction value is fixed at $E(B-V)=0.2$. The results are shown in Fig. 3 where we plot the extinction values derived either from $(U-B)$ or from $(B-V)$ (from the Eqs. (6) and (7), respectively) as a function of mass-loss rate (top panel) or as a function of $\beta$ (bottom panel). From this figure we can draw some important conclusions:

- the wind serves as an additional extinction source ${ }^{1}$ and influences the individual bands differently (see Fig. 1). This means that the resulting color index of a star depends not only on the interstellar extinction, but also on the wind contamination, the latter having a different wavelength dependence than the former;

- whether the observed magnitudes suffer from some circumstellar extinction caused by a strong wind can easily be tested by inspecting at least two color indices, e.g. $B-V$ and $U-B$ as shown here. If they deliver different values for

1 We call it extinction, even though the emission of the wind itself, which adds to the stellar flux, is much stronger than the amount of extinction the star light experiences when passing through the wind zone (see Kraus et al. 2008a). 
Table 1. Sample of galactic early B supergiants from Crowther et al. (2006).

\begin{tabular}{|c|c|c|c|c|c|c|c|c|c|c|c|c|c|}
\hline \# & HD & Name & Sp. Type & $\begin{array}{c}V \\
{[\mathrm{mag}]}\end{array}$ & $\begin{array}{l}B-V \\
{[\mathrm{mag}]}\end{array}$ & D.M. & Ref. & $\begin{array}{l}T_{\text {eff }} \\
{[\mathrm{K}]}\end{array}$ & $\log g$ & $\begin{array}{r}R_{*} \\
{\left[R_{\odot}\right]}\end{array}$ & $\begin{array}{r}v_{\infty} \\
{\left[\mathrm{km} \mathrm{s}^{-1}\right]}\end{array}$ & $\begin{array}{c}\dot{M} \\
{\left[10^{-6} M_{\odot} \mathrm{yr}^{-1}\right]}\end{array}$ & $\beta$ \\
\hline 1 & 30614 & $\alpha$ Cam & O9.5 Ia & 4.29 & +0.02 & 10.0 & $\mathrm{~g}$ & 29000 & 3.00 & 26.0 & 1560 & 5.00 & 1.5 \\
\hline 2 & 37128 & $\epsilon$ Ori & B0 Ia & 1.70 & -0.19 & 7.8 & $\mathrm{a}$ & 27000 & 2.90 & 24.0 & 1910 & 2.50 & 1.5 \\
\hline 3 & 91969 & & B0 Ia & 6.52 & +0.00 & 12.2 & $\mathrm{~d}$ & 27500 & 2.95 & 25.3 & 1470 & 1.00 & 1.5 \\
\hline 4 & 94909 & & B0 Ia & 7.34 & +0.48 & 11.5 & $\mathrm{~g}$ & 27000 & 2.90 & 25.5 & 1050 & 2.00 & 1.5 \\
\hline 5 & 122879 & & B0 Ia & 6.42 & +0.12 & 11.7 & $\mathrm{~g}$ & 28000 & 2.95 & 24.4 & 1620 & 3.00 & 1.5 \\
\hline 6 & 38771 & $\kappa$ Ori & B0.5 Ia & 2.05 & -0.18 & 8.0 & $\mathrm{a}$ & 26500 & 2.90 & 22.2 & 1525 & 0.90 & 1.5 \\
\hline 7 & 115842 & & B0.5 Ia & 6.02 & +0.29 & 11.3 & $\mathrm{~g}$ & 25500 & 2.85 & 34.2 & 1180 & 2.00 & 1.5 \\
\hline 8 & 152234 & & B0.5 Ia & 5.44 & +0.20 & 11.5 & c & 26000 & 2.85 & 42.4 & 1450 & 2.70 & 1.5 \\
\hline 9 & 2905 & $\kappa$ Cas & BC0.7 Ia & 4.16 & +0.14 & 10.2 & $\mathrm{~b}$ & 21500 & 2.60 & 41.4 & 1105 & 2.00 & 2.0 \\
\hline 10 & 91943 & & B0.7 Ia & 6.73 & +0.07 & 12.2 & d & 24500 & 2.80 & 26.3 & 1470 & 0.75 & 1.2 \\
\hline 11 & 152235 & & B0.7 Ia & 6.34 & +0.51 & 11.5 & $\mathrm{c}$ & 23000 & 2.65 & 47.1 & 850 & 1.25 & 1.5 \\
\hline 12 & 154090 & & B0.7 Ia & 4.87 & 26 & 10.2 & $\mathrm{~g}$ & 22500 & 2.65 & 36.0 & 915 & 0.95 & 1.5 \\
\hline 13 & 13854 & & B1 Iab & 6.48 & +0.28 & 11.8 & $\mathrm{e}$ & 21500 & 2.55 & 37.4 & 920 & 0.85 & 2.0 \\
\hline 14 & 91316 & $\rho$ Leo & B1 Iab & 3.85 & -0.14 & 10.5 & $\mathrm{~g}$ & 22000 & 2.55 & 37.4 & 1110 & 0.35 & 1.0 \\
\hline 15 & 148688 & & B1 Ia & 5.33 & +0.33 & 10.5 & $\mathrm{~g}$ & 22000 & 2.60 & 36.7 & 725 & 1.75 & 2.0 \\
\hline 16 & 14956 & & B1.5 Ia & 7.19 & +0.72 & 11.8 & e & 21000 & 2.50 & 50.6 & 500 & 1.00 & 2.0 \\
\hline 17 & 152236 & $\zeta^{1}$ Sco & $\mathrm{B} 1.5 \mathrm{Ia}^{+}$ & 4.73 & +0.48 & 11.5 & $\mathrm{c}$ & 18000 & 2.20 & 112.4 & 390 & 6.00 & 2.0 \\
\hline 18 & 190603 & & $\mathrm{~B} 1.5 \mathrm{Ia}^{+}$ & 5.65 & +0.54 & 10.9 & $\mathrm{~g}$ & 18500 & 2.25 & 59.6 & 485 & 2.50 & 3.0 \\
\hline 19 & 14143 & & B2 Ia & 6.66 & +0.50 & 11.8 & e & 18000 & 2.25 & 52.9 & 645 & 1.05 & 2.0 \\
\hline 20 & 14818 & 10 Per & B2 Ia & 6.25 & +0.30 & 11.8 & $\mathrm{e}$ & 18500 & 2.40 & 46.1 & 565 & 0.55 & 2.0 \\
\hline 21 & 41117 & $\chi^{2}$ Ori & B2 Ia & 4.63 & +0.28 & 10.9 & $\mathrm{~b}$ & 19000 & 2.35 & 61.9 & 510 & 0.90 & 2.0 \\
\hline 22 & 194279 & & B2 Ia & 7.05 & +1.02 & 10.4 & $\mathrm{f}$ & 19000 & 2.30 & 44.7 & 550 & 1.05 & 2.5 \\
\hline 23 & 198478 & 55 Cyg & B2.5 Ia & 4.86 & +0.42 & 9.5 & $\mathrm{f}^{1}$ & 16500 & 2.15 & 40.0 & 470 & 0.23 & 2.0 \\
\hline 24 & 14134 & & B3 Ia & 6.55 & +0.45 & 11.8 & $\mathrm{e}$ & 16000 & 2.05 & 56.7 & 465 & 0.52 & 2.0 \\
\hline 25 & 53138 & $o^{2} \mathrm{CMa}$ & B3 Ia & 3.01 & -0.08 & 10.2 & $\mathrm{~g}$ & 15500 & 2.05 & 65.0 & 865 & 0.36 & 2.0 \\
\hline
\end{tabular}

References to the distance modulus, D.M., are (a) Brown et al. (1993); (b) Blaha \& Humphreys (1989); (c) Baume et al. (1999); (d) Baume et al. (2003); (e) Garmany \& Stencel (1992); (f) Humphreys (1978); (g) Crowther et al. (2006).

${ }^{1}$ The distance modulus for the star HD 198478 is 10.22 mag according to Humphreys (1978). To stay conform with CLW, we will use their value of 9.5 .

the interstellar extinction, then circumstellar extinction due to the wind plays a non-negligible role;

- in the case of a non-negligible wind emission, the $B-V$ color index always overestimates the real interstellar extinction value.

Of course, the strength of the wind contribution not only depends on the mass-loss rate or the $\beta$ parameter, but on the density distribution within the wind continuum emitting region in general. Whether the wind has a significant influence on the photometric values, hence on the color indices, depends, on the one hand, certainly on the wind parameters defining the density distribution, but also, on the other hand, on the stellar parameters determining the strength of the stellar continuum flux.

In the following we, therefore, investigate the wind influence on the color index $B-V$ of a well-studied sample of galactic early-B type supergiants, with the aim of deriving their interstellar exinction parameters again by taking the wind influence at the $B$ and $V$ bands into account.

\section{The B supergiant sample}

We make use of a sample of galactic early-B type supergiants investigated by Crowther et al. (2006, in the following referred to as CLW). Their detailed spectral analysis revealed the complete set of stellar and wind parameters, and interestingly, they found for all (except one) stars of their sample $\beta$ values higher than 1.0. Their sample is therefore ideal to study the influence of the wind emission at optical wavelengths (i.e. especially at the photometric bands $B$ and $V$ ) of early $\mathrm{B}$ supergiants with slowly accelerating winds, i.e. winds with high $\beta$ values.
The sample B supergiants are listed in Table 1. Given are the star number and name, the spectral type and luminosity class, the $V$ and $B$ band magnitudes, and the distance modulus with references. The effective temperature, surface gravity, mass-loss rates, and $\beta$ values have been derived by CLW, and the terminal velocities by Howarth et al. (1997). These values will be used as fixed input into our model calculations. The stellar radii listed in Table 1 have also been derived by CLW. Since we cannot a priori adopt that these radii will not change when accounting for the stellar wind contamination of the photometric data, we use them only in the first step of the wind calculation as the lower boundary of the wind zone.

The effective temperature as given by CLW is independent of reddening. We therefore consider the CLW values given in Table 1 as reasonable. The effective temperature is used further to constrain the electron temperature in the wind. The electron temperature distribution in line-driven winds of early-type stars can be written in the form

$T_{\mathrm{e}}(r)=0.79 T_{\mathrm{eff}}-0.51 \frac{v(r)}{v_{\infty}} T_{\mathrm{eff}}$

as shown by Bunn \& Drew (1992). The generation region of the optical wind continuum is in the close vicinity to the stellar surface, i.e. within less than $2 R_{*}$. The extent of this region is largely independent of the value of $\beta$. In addition, the wind temperature within this region hardly changes for $\beta>1.0$. We, therefore, treat the stellar winds as being isothermal, with $T_{\mathrm{e}}(r)=T_{\mathrm{e}}\left(R_{*}\right)$. As discussed in Kraus et al. (2008a), this delivers a lower limit for the wind emission for winds with $\beta \simeq 1$, while winds with $\beta>1$ are hardly affected by the assumption of isothermality. Since all (except one) stars in the sample have $\beta>1$, the 
assumption of an isothermal wind is therefore a reasonable approximation for our wind calculations.

\section{Results}

In this section we first derive an expression for the color index $\left(B_{*}-V_{*}\right)$ for the radiation emerging from the star based on observed quantities and the knowledge of the interstellar and wind contributions. From a comparison with intrinsic color indices, we then compute the interstellar extinction values towards the stars in our sample.

To calculate the interstellar extinction, $A_{V}$, for the supergiant sample we make use of Eq. (8). This equation can be rewritten to solve for the stellar flux, delivering

$F_{\lambda}^{*}=\left(F_{\lambda} \mathrm{e}^{0.4 \ln (10) \frac{A_{\lambda}}{A_{V}} A_{V}}-F_{\lambda}^{\text {wind }}\right) \mathrm{e}^{\tau_{\lambda}}$.

The first term in parentheses is the observed, extinctioncorrected flux, which results from the observed magnitude, $\operatorname{Mag}_{\lambda}$, at wavelength $\lambda$ via the conversion

$F_{\lambda} \mathrm{e}^{0.4 \ln (10) \frac{A_{\lambda}}{A_{V}} A_{V}}=F_{\text {calib }, \lambda} 10^{-0.4\left(\operatorname{Mag}_{\lambda}-A_{V}\right)}$

where $F_{\text {calib, } \lambda}$ is the calibration flux for this band, corresponding to a star with zero magnitude. Converting the stellar flux given by Eq. (10) into magnitudes and evaluating it for the $B$ and $V$ bands delivers

$B_{*}=B_{\text {wind }}+B_{\text {extinc }}+B_{\text {abs }}$,
$V_{*}=V_{\text {wind }}+V_{\text {extinc }}+V_{\text {abs }}$,

with the following definitions:

$$
\begin{aligned}
B_{\text {wind }} & =-2.5 \log \frac{F_{B}^{\text {wind }}}{F_{B}^{\text {calib }}}, \quad B_{\text {abs }}=-2.5 \log \mathrm{e}^{\tau_{B}}, \\
B_{\text {extinc }} & =-2.5 \log \left(\frac{F_{B}^{\text {calib }}}{F_{B}^{\text {wind }}} 10^{-0.4\left(B-\frac{A_{B}}{A_{V}} A_{V}\right)}-1\right), \\
V_{\text {wind }} & =-2.5 \log \frac{F_{V}^{\text {wind }}}{F_{V}^{\text {calib }}}, \quad V_{\text {abs }}=-2.5 \log \mathrm{e}^{\tau_{V}}, \\
V_{\text {extinc }} & =-2.5 \log \left(\frac{F_{V}^{\text {calib }}}{F_{V}^{\text {wind }}} 10^{-0.4\left(V-A_{V}\right)}-1\right) .
\end{aligned}
$$

Here, $B_{\text {wind }}$ and $V_{\text {wind }}$ are the magnitudes of the wind emission in the $B$ and $V$ band, respectively, $B_{\text {extinc }}$ and $V_{\text {extinc }}$ are terms containing the interstellar extinction, and $B_{\text {abs }}$ and $V_{\text {abs }}$ deal with the absorption of the stellar flux passing through the wind. $B$ and $V$ in the exponents of Eqs. (15) and (17) are the observed $B$ and $V$ band magnitudes, and the parameters $F^{\text {calib }}$ give the absolute flux calibration in the individual bands. The parameter $A_{B} / A_{V}$ in Eq. (15) follows from the mean interstellar extinction curve (see e.g. Cardelli et al. 1989; Mathis 1990). It is worth mentioning that the mean interstellar extinction curve with $R=3.1$ is chosen for being able to compare our results to those of CLW. In fact, when dealing with the accurate extinction determination of individual stars, the application of different interstellar extinction curves might be needed, to account for the differences in the interstellar medium composition along different lines of sight (see, e.g., Fitzpatrick 1999; Fitzpatrick \& Massa 2005).

Since the interstellar extinction $A_{V}$ that appears in the relations Eqs. (15) and (17) is not known a priori, Eqs. (12) and (13) form a coupled set of two equations for three independent variables, $B_{*}, V_{*}$, and $A_{V}$. This system of equations is hence underdetermined and cannot be solved without further constraints. On the other hand, we can make use of the fact that

$\left(B_{*}-V_{*}\right) \equiv(B-V)_{0}$

must be valid, i.e., the $\left(B_{*}-V_{*}\right)$ color index, calculated by our analysis, must agree with the intrinsic $(B-V)_{0}$ color index of a star with the same spectral type and luminosity class. With this additional constrain, the problem is reduced to one single equation with only one unknown parameter, i.e., the interstellar extinction $A_{V}$, which can be solved easily. For calculating the $\left(B_{*}-V_{*}\right)$ color index we do not need our (rather simple) stellar atmosphere code, which only treats $\mathrm{H}$ and $\mathrm{He}$. Instead, this color index follows purely from the set of Eqs. (12) to (17).

To solve Eq. (18), we need to specify the intrinsic color index for each of our sample stars. Since we assume that the effective temperatures listed in Table 1 are correct because they have been derived with a reddening-independent method, the spectral types of the sample stars are fixed (see Table 1), and, following CLW, we apply the intrinsic color indices from the tables of SchmidtKaler (1982).

The wind emission is calculated as described in Sect. 2, i.e. we adopt the wind as unclumped, spherically symmetric, and isothermal. Under these assumptions, the wind emission, $F^{\text {wind }}$, is only a function of the electron temperature and density distribution, $n_{\mathrm{e}}(r)$. For our supergiant sample with effective temperatures in the range $15000 \ldots 30000 \mathrm{~K}$, it is reasonable to assume that hydrogen is fully ionized and helium is singly ionized, while we neglect the contribution of the metals as free electron donators; i.e., the electron density used is a lower limit. With a helium abundance of about $10 \%$, the number density of free electrons in the wind becomes $n_{\mathrm{e}}(r)=1.1 n_{\mathrm{H}}(r)$. The number density of hydrogen, $n_{\mathrm{H}}(r)$, thereby follows from the equation of mass continuity that is proportional to the ratio of mass-loss rate over wind velocity distribution. With the knowledge of the mass-loss rate, $\dot{M}$, and wind velocity distribution given by $v_{\infty}$ and $\beta$ for each star (Table 1), we can then compute the electron density distribution in the wind.

Besides the density distribution, the inner edge of the wind, determined by the stellar radius, plays a crucial role in defining the lower boundary for the wind calculations. Since the wind continuum at optical wavelengths is generated within the innermost $2 R_{*}$, a change in stellar radius will influence the total wind emission directly.

Since we do not know whether the stellar parameters derived by CLW, such as the radii, will change as a result of the wind influence, we use their values as listed in Table 1 as first-guess input parameters for the wind calculations. The interstellar extinction and stellar radius for each star thus has to be calculated iteratively:

1. the stellar radius, $R_{*, \mathrm{CLW}}$, as derived by CLW is used as an input parameter;

2. with the stellar radius and wind parameters fixed, we next calculate the wind emission, $F^{\text {wind }}$, in the $B$ and $V$ bands, as well as the wind optical depths $\tau_{B}$ and $\tau_{V}$, needed to calculate the set of Eqs. (14) and (16);

3. the interstellar extinction $A_{V}$ then follows from solving Eq. (18), and the pure (i.e. interstellar and circumstellar extinction corrected) stellar $B_{*}$ and $V_{*}$ band magnitudes can be calculated from Eqs. (12) and (13), respectively; 


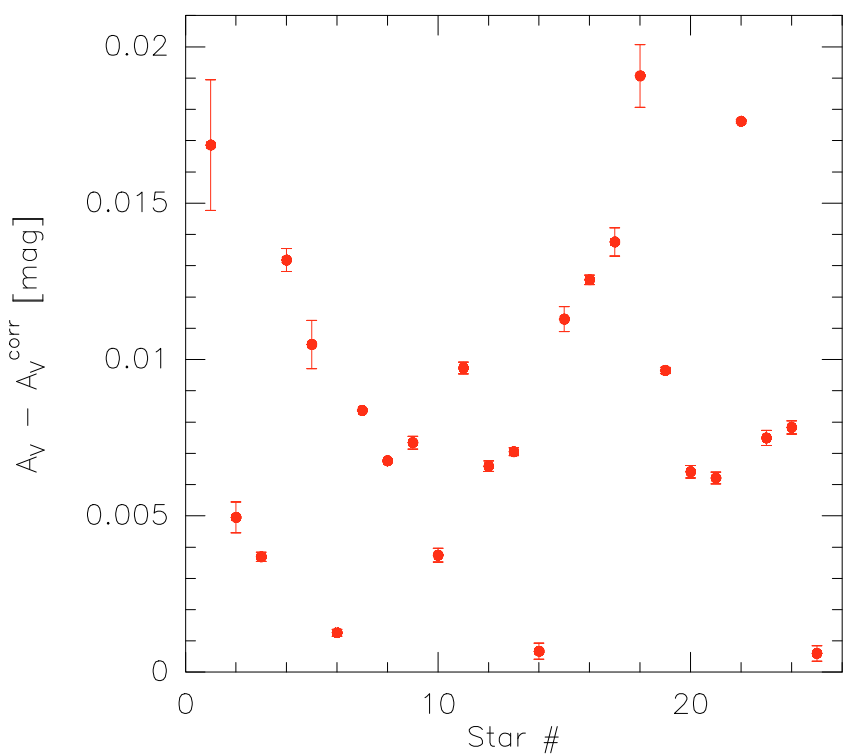

Fig. 4. Difference in interstellar extinction of the supergiant sample calculated from the observed $(B-V)$ color index (labeled $\left.A_{V}\right)$ compared to the value after taking the wind influence into account (labeled $A_{V}^{\text {corr }}$ ). The star number along the $x$-axis corresponds to the numbering in Table 1.

4. the stellar radius can be calculated in terms of the radius derived by CLW. Since we are dealing with two different bands, we obtain two radii given by

$$
\left.R_{*}^{2}\right|_{\mathrm{V}}=R_{*, \mathrm{CLW}}^{2} 10^{-0.4\left(V_{*}-V+A_{V}^{\mathrm{CLW}}\right)}
$$

from the $V$ band flux, and

$$
\left.R_{*}^{2}\right|_{\mathrm{B}}=R_{*, \mathrm{CLW}}^{2} 10^{-0.4\left(B_{*}-B+\frac{A_{B}}{A_{V}} A_{V}^{\mathrm{CLW}}\right)},
$$

from the $B$ band flux. Of course, these two radii must be identical. In these relations, $B$ and $V$ are the observed magnitudes, and $A_{\mathrm{V}}^{\mathrm{CLW}}$ is the interstellar extinction as derived by CLW, based on the intrinsic colors from Schmidt-Kaler (1982);

5. our newly derived stellar radius is then compared with the input radius. If they do not agree, the new radius is chosen as input radius, and the calculations are repeated from step 2 on, until output and input radii agree with an accuracy of better than $0.01 \%$.

The wind influence on the $B$ and $V$ band fluxes cannot be expressed easily in terms of, e.g., the recently introduced windstrength related $Q^{\prime}$ parameter (see Markova \& Puls 2008). In fact, it was not possible to find a unique parameter, which would express the wind influence in a self-consistent way, and against which we could have plotted the deviations in $A_{V}$. The results from our iterative calculations are therefore shown in Fig. 4 versus the star number from Table 1 . For each star within the sample of 25 early-type B supergiants we plotted the difference in extinction, $A_{V}$, derived from the observed and the wind corrected color index $(B-V)$. For all stars, this difference is positive, meaning that the observed color index always overestimates the real interstellar extinction. This overestimation can be as high as $0.02 \mathrm{mag}$.

Also included in Fig. 4 are the error bars, which have been calculated based on the consideration of two main sources of errors: (i) the calibration fluxes from Bessell (1979) for a zero magnitude star, and (ii) the choice of the intrinsic color indices
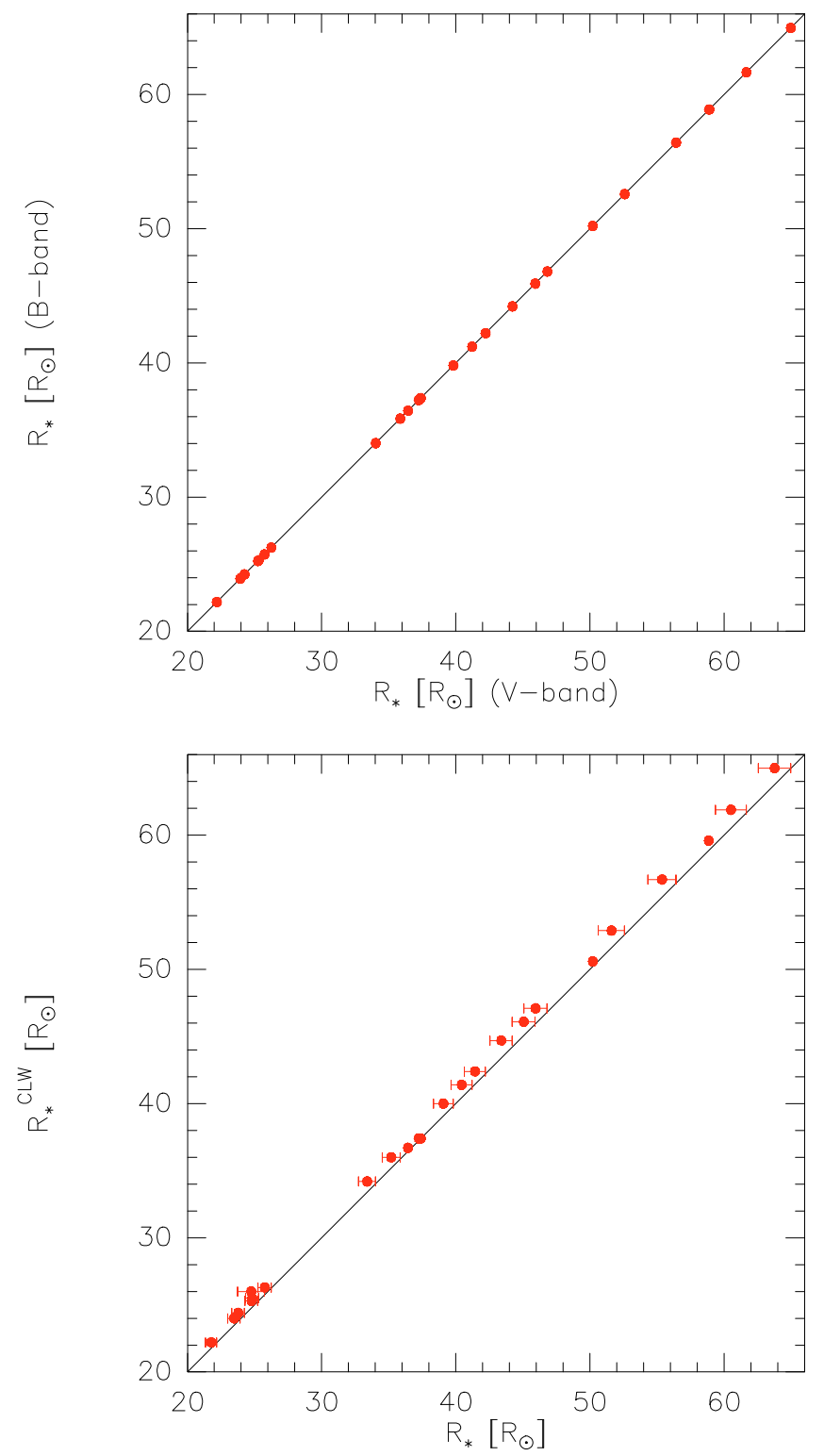

Fig. 5. Final results for the stellar radii (except for the star HD 152236 with $R_{*}>100 R_{\odot}$, which has been skipped in this plot). The top panel shows the excellent agreement between the radii derived in this study from either the $B$ or the $V$ band fluxes. The bottom panel shows our derived radii with error bars based on the erros in $A_{V}$, compared to those of CLW. Our radii are all found to be smaller by up to $6 \%$.

from Schmidt-Kaler (1982). A revision of the zero magnitude for the absolute flux calibration had been suggested by Mégessier (1995), and a new set of zero magnitude calibration fluxes has been calculated, e.g., by Colina et al. (1996). Compared to the old Bessell fluxes, the differences are rather small, with about $3 \%$ for the $B$ band and less than $2 \%$ in the $V$ band. Nevertheless, we adopted this new set of zero magnitude calibration fluxes to estimate the resulting errors in our calculated $A_{V}$ values. We further tested the influence of the chosen set of intrinsic color indices. As mentioned by CLW, their calculations resulted in intrinsic color indices that turned out to be about 0.02 mag more negative than the Schmidt-Kaler values. Our calculations show that, since we are plotting the differences in $A_{V}$, these values are hardly influenced by the possible errors implemented due to differences in the intrinsic color indices. 
The iteration also delivers new results for the stellar radii. These are shown in Fig. 5, where we plot in the top panel the stellar radii resulting from either the $B$ or $V$ band fluxes. They show excellent agreement. When comparing our stellar radii with those of CLW (Fig. 5, bottom panel), it is obvious that we find smaller (or about equal) stellar radii for all supergiants in the sample. The differences in radii can reach up to $6 \%$, where the error bars shown are calculated based on the errors in $A_{V}$.

\section{Discussion}

\subsection{Wind contribution and importance}

The deviations in $A_{V}$ found from our analysis spread from $0.001 \mathrm{mag}$ to $0.02 \mathrm{mag}$ (see Fig. 4). Of course, a deviation of only 0.001 mag cannot be detected easily from the observed colors, since it is within the error bars. Deviations greater than $0.005 \mathrm{mag}$, however, are considered as meaningful. For those stars, we would argue that the wind shows a non-negligible influence on the $(B-V)$ color index.

The deviation in $A_{V}$ is highest for the stars \# 1, 18, and 22. Inspection of their parameters in Table 1 shows that \# 18 is the one with $\beta=3.0$, and $\# 22$ with $\beta=2.5$, i.e., these are the stars with the highest $\beta$ value in the sample. For star \# 1 we see that it has a rather high mass-loss rate. It is therefore not surprising that the overestimation in $A_{V}$ is found to be highest for these three targets.

On the other hand, there are a few targets with almost no deviation in $A_{V}$ (i.e. a deviation of less than $0.005 \mathrm{mag}$ ). These are the stars with lowest $\beta$ values (stars \# 14 and 10 with $\beta=$ 1.0 and 1.2, respectively), as well as some stars with rather low mass-loss rates. But before we can draw any conclusion from this behavior, we need to have a closer look at the wind influence.

A quantitative representation of the wind influence is displayed in Fig. 6. The top panel shows the ratio of the gain in wind emission at the $B$ and $V$ bands over the loss of stellar emission due to the wind extinction. For all stars, and therefore almost independent of the individual stellar and wind parameters, this ratio is found to be about two. This means that the wind emits about twice as much as it absorbs from the stellar light, adding some net amount to the total emission (opposite than the effect of interstellar extinction). In addition, the ratio is always higher in the $V$ band. The wind thus has a stronger influence in the form of net emission in the $V$ band than in the $B$ band, and this difference in wind influence at the two different wavelengths even increases with decreasing effective temperature of the star (i.e. for the stars with higher numbers).

Whether this wind emission will have observable consequences also depends on the continuum strength of the underlying star. If the wind is negligible, then

$\frac{F_{v}^{\text {wind }}}{F_{v}^{*} \mathrm{e}^{-\tau_{v}}} \ll 1$

should be valid. We calculate this ratio for each target of our sample and plot it in the lower panel of Fig. 6. This ratio is found to spread over several orders of magnitude. Obviously, stars \# 14 and 25 show the least wind influence, while stars \# 1 and 18 display the strongest influence, in agreement with their least and strongest deviations in $A_{V}$. For the rest of the sample, the situation is not as clear.

But obviously, a high mass-loss rate or a high $\beta$ value alone is no guarantee of a strong wind influence. The situation is more complicated since, on the one hand, other parameters like the

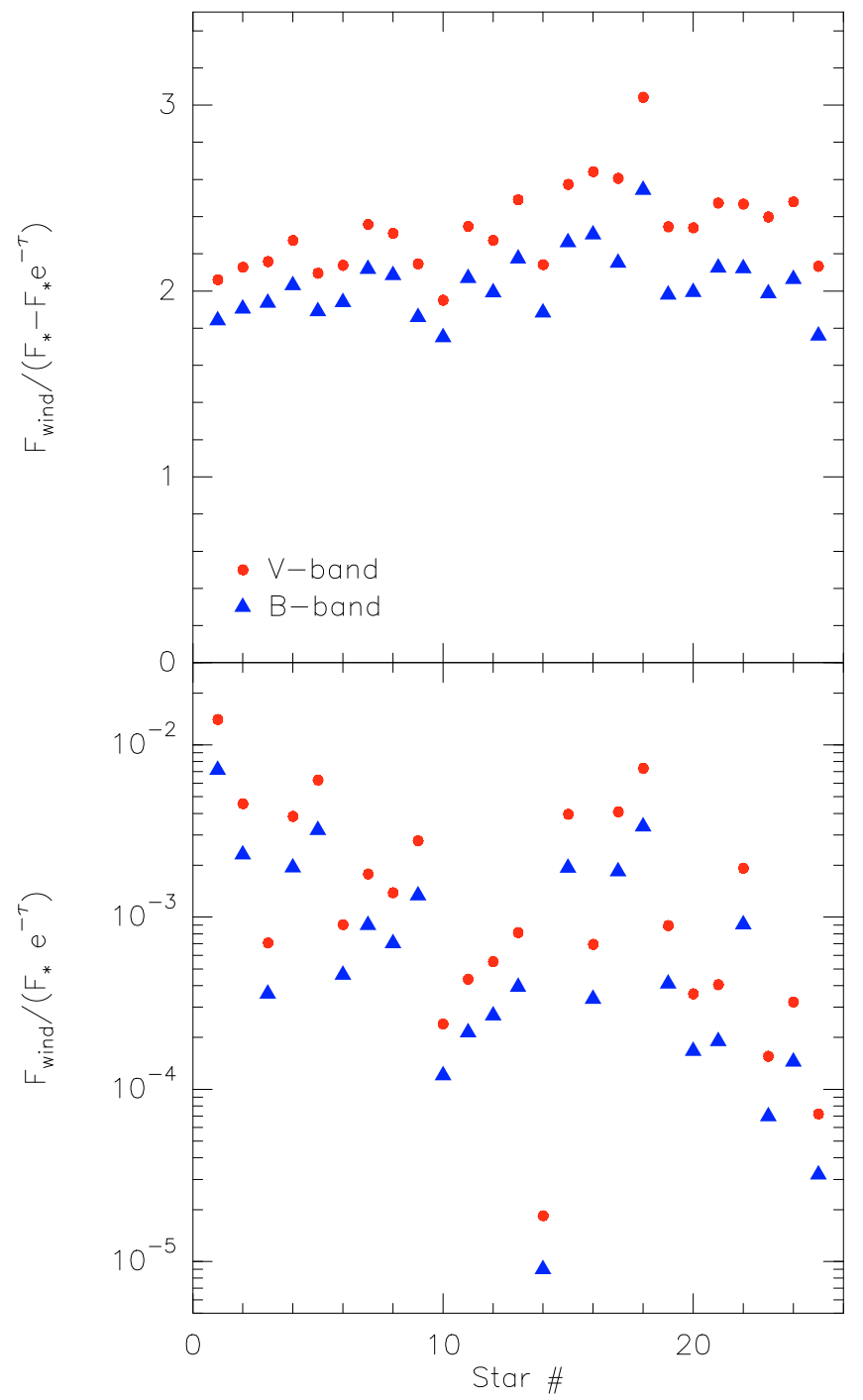

Fig. 6. Top panel: ratio of gain in thermal wind emission over the loss in stellar flux due to the extinction by the wind zone. Bottom panel: ratio of the wind continuum emission compared to the stellar continuum emission.

stellar radius, the wind terminal velocity, as well as the electron temperature, influence the density distribution in the windforming region and the wind emission. On the other hand, the strength of the stellar continuum, which is determined by the effective temperature, stellar radius, and surface gravity also plays a non-negligible role (see bottom panel of Fig. 6).

Our research cannot provide a recipe for which parameter configuration the wind starts to influence the total continuum emission, but we would like to draw attention to its possible (and sometimes rather strong) influence. Whether or not the wind really influences the total continuum has to be checked for each star individually.

\subsection{Reliability of the wind calculations}

The wind calculations were performed with the code of Kraus et al. (2008a). As has been listed already in Sect. 2, this code makes use of several assumptions and simplifications, to keep the computations as simple as possible. However, all these assumptions and simplifications are such that the computed wind emission is a lower limit. This means that, in reality, the 


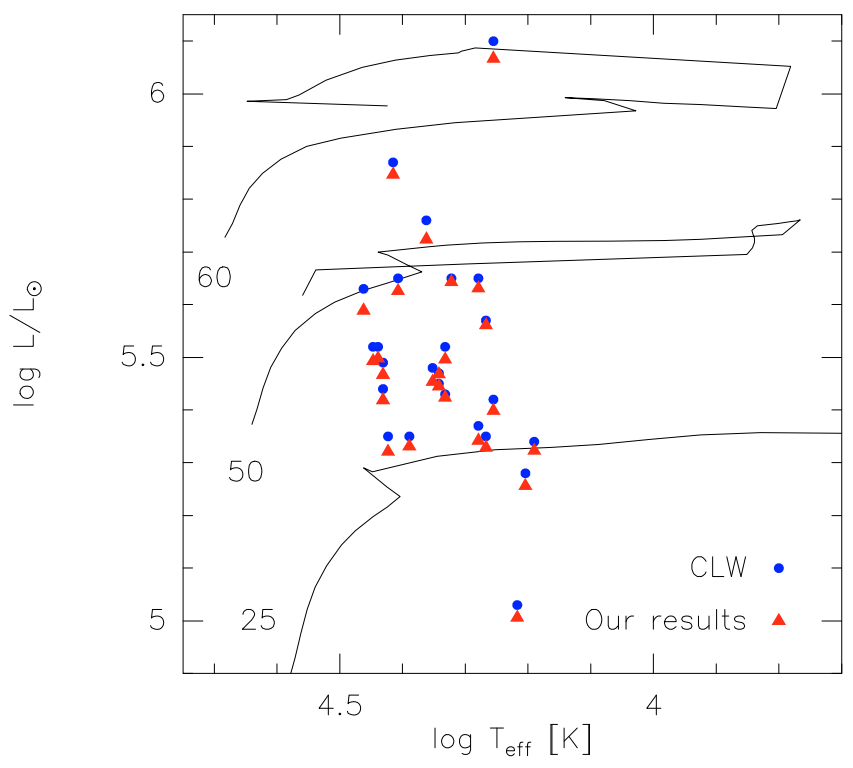

Fig. 7. Location of the supergiant sample in the HR diagram. Shown are our upper limits in luminosities, derived by taking the wind as an extinction source into account, compared to the luminosities determined by CLW. Evolutionary tracks are from Schaller et al. (1992) for nonrotating stars at solar metallicity.

influence of the wind on the photometric data will be even more pronounced than predicted with our model. Consequently, the deviations in $A_{V}$ will be greater, and the resulting stellar radii will be even smaller than those found from our simple calculations. Of course, we cannot quantify the effect. This is only possible with an exact calculation of the emission of the star plus wind system. However, we expect the effects to be noticeable.

\subsection{Consequences of the wind influence}

Besides the overestimation of the interstellar extinction values, the neglect of the wind's influence on the observable continuum emission has further consequences for the set of stellar parameters.

First of all, we found that the stellar radii are smaller. Even though this is an effect of only a few percent, it nevertheless influences other parameters like, e.g., the stellar luminosity. In Sect. 2 we mentioned that a star with a high-density wind appears brighter in the $V$ band (see Fig. 1), so that the real stellar luminosity will be overestimated. With the effective temperatures of the target stars from Table 1 and our revised stellar radii, we calculate the resulting stellar luminosities, which consequently are all lower than the values of CLW. The highest deviation with about $10 \%$ is thereby found for the star \# 1. Even though it is known that the wind strengths tend to increase with luminosities, we do not find any correlation between the amount of luminosity reduction for the sample stars and their luminosities. The reason for this is that the wind influence is not a simple function of the wind strength, but depends on many different stellar and wind parameters, as discussed earlier. Therefore, we cannot make any predictions about its possible influence, but a detailed calculation is necessary for every individual star, checking for the possible wind contamination and hence the expected reduction in luminosity.

The positions of the stars with their revised (i.e. lower) luminosities are shown in Fig. 7. The shift in luminosity (which is likewise a downwards correction of the stellar masses when compared to the evolutionary tracks of Schaller et al. 1992) is quite obvious, and might even increase. Our derived luminosities must therefore be considered as upper limits. The neglect of the wind's influence on the optical continuum thus not only leads to an overestimation of the interstellar extinction, but also to an overestimation of the stellar luminosities and masses.

\subsection{Clumped winds}

Recent results from both observational and theoretical investigations of hot star winds suggest that the winds might be clumped (e.g., Crowther et al. 2002; Hillier et al. 2003; Bouret et al. 2003, 2005). As a consequence of such wind clumping, the mass-loss rates of the stars might be overestimated by about a factor of 3. The mass-loss rates provided by CLW for the OB supergiant sample studied here have been derived under the assumption of smooth rather than clumped winds. Consequently, they might well be lower, resulting in lower wind densities for the sample stars.

A detailed study of the influence of wind clumping is beyond the scope of this paper, since it is not known whether the winds of the stars in our sample are indeed clumped. In a recent study, Kraus et al. (2008b) investigated the influence of wind clumping on the total continuum emission of hot stars with high $\beta$ winds. Using the filling factor approach introduced by Hillier et al. (2003), they found that the emission of clumped winds is slightly reduced compared to unclumped winds. Nevertheless, the winds still contaminate the optical continuum emission. The influence on the total continuum emission when clumping is taken into account will thus be weaker, but the wind contamination will nevertheless be present, even though in a milder form.

However, whether the winds of the studied galactic OB supergiant sample are indeed clumped or whether the high $\beta$ values found by CLW have a completely different cause, needs to be studied in more detail.

\section{Conclusions}

We investigated the influence of winds with high $\beta$ values on the observed photometric fluxes. We focused especially on the problem of proper interstellar extinction derivation from the observed $(B-V)$ color index. For this, we first calculated the wind emission and absorption for a model star plus wind system, increasing the mass-loss rate and the $\beta$ parameter. We found that the wind influences the emission at the individual photometric bands differently, i.e. with a different wavelength dependence than the interstellar extinction does; consequently, any wind contribution might be easily checked by looking at least at two different color indices. We also found that the interstellar extinction derived from the observed $(B-V)$ color index is always overestimated.

We then investigated a sample of galactic early-B type supergiants that has previously been studied in great detail by Crowther et al. (2006). This sample was ideal for our purposes, because for all except one star, Crowther et al. (2006) derived $\beta$ parameters considerably greater than unity. From their $\beta$ parameters and mass-loss rates, we calculated the wind contribution to the $B$ and $V$ band fluxes for all target stars and derived the interstellar extinction values again. We found that, for all stars in the sample, the interstellar extinction is overestimated when it is derived purely from the observed $(B-V)$ color index, without taking the wind's influence into account.

Another consequence of our analysis is that we could derive smaller stellar radii, hence lower luminosities and masses 
for all stars of the supergiant sample. This raises the question of whether the luminosities for all supergiant stars have to be corrected downwards. Even though the absolute values found from our analysis do not exceed about $10 \%$, we want to stress that our wind calculations are only suitable to determine the lower limits of the wind emission. The real downward corrections in stellar luminosities and masses might therefore be higher.

Acknowledgements. We thank our anonymous referees for many constructive suggestions on the original manuscript. M.K. acknowledges financial support from GA AV ČR grant number KJB300030701, and J.K. and M.K. from GA ČR number 205/08/0003. M.B.F. acknowledges financial support from the Belgian Federal Science Policy Office (Research Fellowship for non-EU Postdocs) and from the Centre National de la Recherche Scientifique (CNRS).

\section{References}

Baume, G., Vázquez, R. A., \& Feinstein, A. 1999, A\&AS, 137, 233

Baume, G., Vázquez, R. A., Carraro, G., \& Feinstein, A. 2003, A\&A, 402, 549

Bessell, M. S. 1979, PASP, 91, 589

Blaha, C., \& Humphreys, R. M. 1989, AJ, 98, 1598

Bouret, J.-C., Lanz, T., Hillier, D. J., et al. 2003, ApJ, 595, 1182

Bouret, J.-C., Lanz, T., \& Hillier, D. J. 2005, A\&A, 438, 301

Brown, A. G. A., de Geus, E. J., \& de Zeeuw, P. T. 1994, A\&A, 289, 101

Bunn, J. C., \& Drew, J. E. 1992, MNRAS, 255, 449

Cardelli, J. A., Clayton, G. C., \& Mathis, J. S. 1989, ApJ, 345, 245

Colina, L., Bohlin, R., \& Castelli, F. 1996, Instrument Science Report CAL/SCS008
Crowther, P. A., Hillier, D. J., Evans, C. J., et al. 2002, ApJ, 579, 774

Crowther, P. A., Lennon, D. J., \& Walborn, N. R. 2006, A\&A, 446, 279 (CLW) Evans, C. J., Crowther, P. A., Fullerton, A. W., \& Hillier, D. J. 2004, ApJ, 610, 1021

Fitzpatrick, E. L. 1999, PASP, 111, 63

Fitzpatrick, E. L., \& Massa, D. 2005, AJ, 130, 1127

Garmany, C. D., \& Stencel, R. E. 1992, A\&AS, 94, 211

Hillier, D. J., Lanz, T., Heap, S. R., et al. 2003, ApJ, 588, 1039

Howarth, I. D., Siebert, K. W., Hussain, G. H. J., \& Prinja, R. K. 1997, MNRAS, 284,265

Humphreys, R. M. 1978, ApJS, 38, 309

Kraus, M., Kubát, J., \& Krtička, J. 2008a, A\&A, 481, 499

Kraus, M., Kubát, J., \& Krtička, J. 2008b, in Clumping in Hot Star Winds, ed. W.-R. Hamann, A. Feldmeier, \& L. Oskinova (Potsdam: Univ.-Verl.), 51

Kraus, M., Borges Fernandes, M., Kubát, J., \& de Araújo, F. X. 2008c, A\&A, 487, 697

Kubát, J. 2003, in Modelling of Stellar Atmospheres, ed. N. Piskunov, W. W. Weiss, \& D. F. Gray (San Francisco: ASP), IAU Symp., 210, A8

Leitherer, C., \& Wolf, B. 1984, A\&A, 132, 151

Markova, N., \& Puls, J. 2008, A\&A, 478, 823

Martins, F., Schaerer, D., \& Hillier, D. J. 2005, A\&A, 436, 1049

Mathis, J. S. 1990, ARA\&A, 28, 37

Mégessier, C. 1995, A\&A, 296, 771

Mokiem, M. R., de Koter, A., Vink, J. S., et al. 2007, A\&A, 473, 603

Schaller, G., Schaerer, D., Meynet, G., \& Maeder, A. 1992, A\&AS, 96, 269

Schmidt-Kaler, Th. 1982, in Landolt-Börnstein, New Series, Group IV, Vol. 2b, ed. K. Schaifers, \& H. H. Voigt (Berlin: Springer), 1

Trundle, C., \& Lennon, D. J. 2005, A\&A, 434, 677

Trundle, C., Lennon, D. J., Puls, J., \& Dufton, P. L. 2004, A\&A, 417, 217

Waters, L. B. F. M., \& Lamers, H. J. G. L. M. 1984, A\&AS, 57, 327 